\title{
Impact of treating chronic hepatitis $C$ infection with direct-acting antivirals on the risk of hepatocellular carcinoma recurrence
}

\author{
Nevine Ibrahim Musa*, Inas Elkhedr Mohamed and Ahmed Samir Abohalima
}

\begin{abstract}
Background: The impact of direct-acting antivirals (DAAs) remains a debate, whether they accelerate the recurrence rate of hepatitis $\mathrm{C}$ virus (HCV)-related hepatocellular carcinoma (HCC) after curative therapy. We evaluated the impact of direct-acting antiviral therapy on the rate of recurrence of HCV-related hepatocellular carcinoma following intervention in Egyptian patients.

Results: The results of the study represented an HCC recurrence rate of 38\% in patients who received direct-acting antiviral therapy after HCC intervention versus $62 \%$ in those who did not receive antiviral therapy. In group I, according to the Barcelona Clinic of Liver Cancer (BCLC) staging, a higher recurrence rate was observed (57.9\%) among patients who were classified as BCLC stage B.

Conclusions: HCC patients who did not receive direct-acting antiviral therapy after HCC intervention had a greater risk of HCC recurrence. DAAs did not increase the risk of HCC recurrence following HCC treatment; however, it did not abolish it. Close monitoring of patients after antiviral therapy is recommended.
\end{abstract}

Keywords: Hepatocellular carcinoma, Direct-acting antivirals, Hepatitis C virus

\section{Background}

Liver cirrhosis is the most crucial risk factor for hepatocellular carcinoma (HCC) [1]. Moreover, hepatitis C virus $(\mathrm{HCV})$ infection is a significant cause in the development of cirrhosis in the USA, Europe, and other countries like Egypt [2, 3]. HCV infects about $2-2.5 \%$ of the world's population (130-150 million people) according to WHO. The prevalence of $\mathrm{HCV}$ in Egypt is about 12\% among males and $8 \%$ among females [4]. Hepatocarcinogenesis risk in $\mathrm{HCV}$-infected cases with advanced cirrhosis varies from 2 to $8 \%$ per year. One of the lessons learned during the era of interferon (IFN)-based therapy for $\mathrm{HCV}$ was that eradication of $\mathrm{HCV}$ reduced the risk of developing HCC, regardless of the degree of hepatic fibrosis [5]. In addition, patients with previously ablated HCC who achieved a sustained virological response (SVR) due to

\footnotetext{
* Correspondence: nevine_musa@yahoo.com

Department of Internal Medicine, Ain Shams University, Cairo 11341, Egypt
}

IFN-based therapy had a better prognosis than those who did not [6]. Achieving SVR is the single most important factor predicting a lower risk of developing $\mathrm{HCV}$-induced HCC, providing a $20 \%$ reduction rate in the incidence of HCC [7]. Notably, IFN-based therapy was limited to patients without advanced cirrhosis. The introduction of highly effective direct-acting antivirals (DAAs) was conventionally expected by practicing hepatologists to lead to the extension of this benefit to all patients, including those who were not candidates for IFN-based therapy [8]. DAAs directly inhibit the HCV replication cycle leading to dramatic improvement and a higher SVR than the previously used interferon-based regimens [9]. HCC occurrence is defined as a new appearance of HCC in patients with no history of liver tumor, while HCC recurrence is defined as a reappearance of $\mathrm{HCC}$ in patients who had a previous successful radical therapy for HCC. However, the clinical experience of using DAAs has resulted in a major debate with important clinical implications regarding the 
relationship between DAAs and the development of HCC. Many authors suggested a link between the use of DAAs and the occurrence of $\mathrm{HCC}$, while others insisted that the use of DAAs is protective against HCC development [10].

This study aimed to evaluate the impact of DAAs on the recurrence rate of HCV-related HCC by 6 months or more following intervention in Egyptian patients.

\section{Methods}

\section{Subjects}

This retrospective study was carried out on two groups of patients recruited from the Hepatology and Gastroenterology Unit (Internal Medicine Department), Ain Shams University (ASU) Hospital, and from the Center For Treatment of Viral Hepatitis at Ain Shams University (one of the National Committee for the Control of Viral Hepatitis $(\mathrm{NCCVH})$ centers in Cairo) from August 2016 to April 2018. Eligible patients were male and non-pregnant female patients, aged 18-75 years, with chronic HCV infection and positive HCV RNA by PCR. One hundred patients were included and assigned into two groups.

1. Group I included 50 patients who received DAAs for chronic HCV infection after the management of HCC. Patients were followed up for a period of 1 year for any HCC recurrence.

2. Group II included 50 patients with $\mathrm{HCV}$-associated HCC who received management for HCC and not treated with DAAs and also followed up for 1 year for HCC recurrence, and treatment-naive or treatment-experienced patients with HCV (defined as patients who experienced virological failure after receiving a previous course of PegIFN or sofosbuvir-containing regimen).

All patients had compensated Child A or B cirrhosis according to the Child-Pugh classification [11]. Diagnosis of cirrhosis based on clinical, laboratory, and radiological study confirmed by FibroScan showed liver stiffness measurement more than or equal to $14.5 \mathrm{kPa}$ [12].

We apply various exclusion criteria during the selection of the population of the study: decompensated liver, HCC (except after 6 months of intervention aiming at a cure with no evidence of activity by dynamic imaging triphasic computed tomography or MRI), extra-hepatic malignancies (at least after 2 years of a disease-free interval), HBV co-infected patients, lactation or inability to use an effective contraceptive method, and patients with evidence of other causes of liver diseases, including hepatitis A, hepatitis B, autoimmune hepatitis, alcoholic liver disease, drug-induced hepatitis, hemochromatosis, Wilson's disease, or $\alpha 1$-antitrypsin deficiency.

The exclusion criteria for the administration of DAAs included (according to NCCVH protocol) the following: total bilirubin more than $3 \mathrm{mg} / \mathrm{dl}$, serum albumin less than $2.8 \mathrm{~g} / \mathrm{dl}$, international normalization ratio [INR] more than 1.7, platelet count less than $50,000 / \mathrm{mm}^{3}$, patients with any advanced systemic disease that could affect liver disease progression and the choice of antiviral regimen, and pregnancy or inability to use effective contraception method in women.

\section{Methods}

According to the National Committee for the Control of Viral Hepatitis Egyptian Treatment Protocol, all participants were subjected to the following:

1. Detailed history taking and full clinical examination.

2. Laboratory investigations at baseline:

(a) Complete blood count (CBC), liver profile (aspartate aminotransferase [AST], alanine aminotransferase $[\mathrm{ALT}]$, total and direct bilirubin, S. albumin), S. creatinine, prothrombin time, INR, alpha-fetoprotein (AFP), fasting blood sugar, and $\mathrm{HbA1c}$ in diabetics

(b) Hepatitis viral markers: hepatitis B surface antigen and HCV antibody using thirdgeneration ELISA test and pregnancy test for females in the childbearing period

(c) Abdominal ultrasonography: special stress on liver echogenicity and size, portal vein diameter, presence of any hepatic focal lesion (HFL), and splenomegaly

(d) ECG for males above 40 years and females above 50 years and echocardiography for patients above 60 years

3. Transient elastography: FibroScan (M probe; Echosens, Touch, Paris) was carried out by an experienced examiner in all patients, as a pretreatment assessment. During the examination, the patient was lying in a dorsal decubitus position with the right arm in maximal abduction to enlarge the intercostal space in which the probe was placed. The median liver stiffness of 10 successful measurements fulfilling the criteria was noted in kilopascal.

4. Laboratory investigations at follow-up visits (every 4 weeks): CBC, AST, ALT, INR, and total bilirubin.

(a) Serum HCV RNA level was measured before treatment, at the end of treatment (12 weeks and 24 weeks), and at 12 weeks after completion of treatment

(b) Sustained virological response (SVR12) defined as undetectable HCV RNA 12 weeks after completion of therapy by a sensitive HCV RNA assay [13].

5. We evaluated the following parameters: 
(a) Recurrence: percentage of patients who had a detectable viral load by PCR 12 weeks after the end of treatment.

(b) Response rate: the percentage of patients who achieved SVR12.

\section{Management of HCC}

Patients with proved HCC were co-managed with the interventional radiology team according to the regimen advocated by the Barcelona Clinic of Liver Cancer (BCLC) and selected carefully after expert opinion. Patients who underwent radiological intervention, surgical resection, and liver transplantation were enrolled for DAAs after at least 6 months of follow-up to exclude evidence of residual tumor activity and or early detection of any HFL, by dynamic imaging triphasic computed tomography or MRI and AFP.

HCC recurrence is defined as any confirmed intra- or extrahepatic HCC lesions detected by radiographic or histopathological diagnostics after treatment. In the present study, we depend on imaging techniques and standard follow-up including abdominal multislice imaging (CT or MRI scans) as mentioned before [14-16].

The study was performed in accordance with ethical standards. Faculty of Medicine, Ain Shams University Ethical Committee approval was taken before starting the study, and the study protocol conforms to the ethical guidelines of the 1975 Declaration of Helsinki.

\section{Statistical analysis}

Data were fed to the computer and analyzed using the IBM SPSS software package version 20.0. (Armonk, NY: IBM Corp.). Qualitative data were described using number and percent. The Kolmogorov-Smirnov test was used to verify the normality of distribution. Quantitative data were described using range (minimum and maximum), mean, standard deviation, and median. The used tests were chisquare test, Fisher's exact or Monte Carlo correction, Student $t$ test, and Mann Whitney test. The significance of the obtained results was judged at the $5 \%$ level.

\section{Results}

Among 100 patients with chronic HCV infection in the final analyses, $42(84 \%)$ males and $8(16 \%)$ females were included in group I, and $36(72 \%)$ males and $14(28 \%)$ females in group II. The mean age in group I was $60.48 \pm$ 8.15 and $60.86 \pm 9.47$ in group II without a significant statistical difference $(p=0.83)$.

In studying the Child-Pugh classification, $62 \%$ of patients in group I were Child A and $38 \%$ were Child B, while $52 \%$ of patients in group II were Child A and $48 \%$ were Child $\mathrm{B}$ without a significant difference $(p$ value $=$ 0.313).
Descriptive analysis of different DAA treatment regimens received by patients in group I

Fifty patients were treated with DAA, 30 patients received sofosbuvir/daclatasvir and ribavirin, and 9 patients received sofosbuvir and ribavirin, and other regimens are described in Table 1.

\section{Comparing different HCC treatment modalities between groups I and II}

In group I, 11 patients underwent hepatic resection, 27 patients underwent radiofrequency ablation (RFA), $10 \mathrm{pa}-$ tients treated with LDLT, and two patients treated with alcohol injection, while in group II, 12 patients underwent hepatic resection, 35 patients underwent RFA, and 3 patients treated with alcohol injection (Table 2).

\section{HCC recurrence rates in both groups}

The recurrence rate was significantly higher in group II with a $p$ value of 0.016 (Table 3 ).

\section{Relation between HCC recurrence and parameters in group I}

There is a significant relation between $\mathrm{HCC}$ recurrence and $\mathrm{BCLC}$ stage $(p$ value $=0.004)$, where BCLC stage $\mathrm{B}$ was associated with a higher recurrence rate $(57.9 \%$ of patients who developed recurrence were stage B) (Table 4).

\section{Relation between HCC recurrence and parameters in group II}

There is a significant relation between recurrence and HCC treatment modality ( $p$ value for hepatectomy = 0.049), while the number, size of focal lesions, and BCLC stage did not affect HCC recurrence (Table 5).

Table 1 Descriptive analysis of different DAA treatment regimens received by patients in group I

\begin{tabular}{lll}
\hline HCV treatment & Number & Percent \\
\hline SOF/DAC & 1 & 2.0 \\
SOF/DAC/RBV & 30 & 60.0 \\
SOF/LED & 5 & 10.0 \\
SOF/LED/RBV & 1 & 2.0 \\
SOF/RBV & 9 & 18.0 \\
SOF/SIM & 4 & 8.0 \\
HCV treatment (weeks) & & \\
$\quad$ Min.-Max. & $12.0-24.0$ & \\
$\quad$ Mean \pm SD & $14.64 \pm 5.02$ & \\
$\quad$ Median & 12.0 & 84.0 \\
PCR after TIT & & 16.0 \\
$\quad$ SVR & 42 & \\
$\quad$ Relapser & 8 & \\
SOF sofosbuvir, LED ledipasvir, $R B V$ ribavirin, SIM simeprevir, DAC daclatasvir, \\
PCR polymerase chain reaction, SVR sustained virological response
\end{tabular}


Table 2 Comparison between the two studied groups according to HCC treatment

\begin{tabular}{|c|c|c|c|c|}
\hline HCC treatment modalities & Group I ( $(n=50)$, no. (\%) & Group II $(n=50)$, no. $(\%)$ & $x^{2}$ & $p$ \\
\hline Hepatic resection & $11(22 \%)$ & $12(24 \%)$ & 1.002 & 0.182 \\
\hline RFA & $27(54 \%)$ & $35(70 \%)$ & 3.549 & 0.420 \\
\hline LDLT & $10(20 \%)$ & $0(0.0 \%)$ & $7.696^{*}$ & $p=0.006^{*}$ \\
\hline Alcohol injection & $2(4.0 \%)$ & $3(6.0 \%)$ & 1.010 & $p=1.000$ \\
\hline
\end{tabular}

RFA radiofrequency ablation, $L D L T$ living donor liver transplant

*Statistically significant at $p \leq 0.05$

\section{HCC recurrence rate and time after starting DAAs in group I}

The recurrence rate in group I was 38\% (19 patients out of 50) with a median recurrence time of 8 months after starting antiviral therapy (Table 6).

\section{$\mathrm{HCC}$ recurrence rate and time after $\mathrm{HCC}$ intervention in group II}

Recurrence rate in group II was $62 \%$ (31 patients out of 50) with a median recurrence time of 3 months after HCC intervention (Table 7)

\section{Discussion}

$\mathrm{HCC}$ is one of the serious complications of chronic $\mathrm{HCV}$ infection, and the risk is increased with advancing hepatic fibrosis and cirrhosis reaching an incidence of about $3.5 \%$ in cirrhotic patients per year [10]. Chronic $\mathrm{HCV}$ infection induces inflammation with consequent hepatocarcinogenesis, so the resolution of $\mathrm{HCV}$ infection should result in a reduced incidence of HCC [17]. HCV treatment outcomes significantly improved after the introduction of new DAAs in the past few years with a response of $>90 \%$ of patients achieving an SVR after 12 weeks of starting treatment [18]. The increased success in $\mathrm{HCV}$ treatment has raised the hope in a significant decrease in the rate of HCC occurrence and even its recurrence after treatment of neoplastic lesions [10]. However, the impact of DAA-based treatment on the incidence of hepatocellular carcinoma (HCC) in patients with cirrhosis and particularly on the incidence of HCC recurrence after successful curative treatment has emerged as a controversial issue with potential clinical implications [19].

Previous studies had conflicting results about the effect of DAA on HCC development or recurrence. This study was performed to highlight this effect specially on

Table 3 Comparison between groups I and II according to HCC recurrence

\begin{tabular}{lllll}
\hline & $\begin{array}{l}\text { Group I }(n=50), \\
\text { no. (\%) }\end{array}$ & $\begin{array}{l}\text { Group II }(n=50), \\
\text { no. (\%) }\end{array}$ & $x^{2}$ & $p$ \\
\hline $\begin{array}{l}\text { HCC } \\
\text { recurrence }\end{array}$ & $19(38 \%)$ & $31(62 \%)$ & $5.760^{*}$ & $0.016^{*}$ \\
\hline
\end{tabular}

*Statistically significant at $p \leq 0.05$
Egyptian patients. The study was carried out on 50 patients who previously received $\mathrm{HCC}$ intervention and treated for HCV using DAAs after confirming HCC regression and response to different treatment modalities. Patients were followed up for at least 1 year after antiviral therapy. We have selected a control group of 50 patients with cured HCC who did not receive DAA therapy, to compare the recurrence rate in both groups and its relation to the antiviral therapy.

The current study addressed an insignificant difference between both groups of patients in demographic data and Child score. Patients in both groups were treated for $\mathrm{HCC}$ with RFA, resection, and alcohol ablation. In group I, ten patients underwent liver transplantation which was not recommended in the second group owing to the universal recurrence of $\mathrm{HCV}$ infection post-liver transplant that should be treated to avoid allograft cirrhosis [20].

Unlike previous studies which raised the concern that DAA therapy may increase or accelerate the risk of HCC recurrence and lead to more aggressive tumors, the results of our study came different. This study identified lower HCC recurrence rates in patients who received antiviral therapy as compared to those who did not. A recurrence rate of $38 \%$ was reported in patients who received antiviral therapy in a median time of 8 months after starting DAA therapy, whereas $62 \%$ of patients who did not receive DAAs developed $\mathrm{HCC}$ recurrence in a median time of 3 months after HCC intervention. These data were not consistent with Reig et al. [19], who demonstrated an HCC recurrence rate of $27.6 \%$ in 58 DAAtreated patients included in their study. This result was significantly higher than that of the non-treated patients, supported by their observations of other studies.

Conti et al. introduced results that matched with Reig et al., concerning $\mathrm{HCC}$ recurrence rates where they demonstrated a recurrence rate of $28.8 \%$ in 59 DAAtreated patients during 24 weeks of post-treatment follow-up. This study lacked a control arm to identify whether this rate differed from the non-treated patients or not [10]. Although the results of our study showed a larger recurrence rate compared to the previous two studies, yet in comparison to the control group, DAAs did not appear to increase HCC recurrence rates. 
Table 4 Relation between HCC recurrence and different parameters in group I

\begin{tabular}{|c|c|c|c|c|}
\hline & \multicolumn{2}{|l|}{ HCC recurrence } & \multirow[t]{2}{*}{ Test of Sig. } & \multirow[t]{2}{*}{$p$} \\
\hline & No $(n=31)$, no. $(\%)$ & Yes $(n=19)$, no. $(\%)$ & & \\
\hline \multicolumn{5}{|l|}{$\mathrm{BCLC}$} \\
\hline 0 & $5(16.1 \%)$ & $0(0.0 \%)$ & $x^{2}=0.161^{*}$ & $0.004^{*}$ \\
\hline A & $21(67.7 \%)$ & $8(42.1 \%)$ & & \\
\hline B & $5(16.1 \%)$ & $11(57.9 \%)$ & & \\
\hline \multicolumn{5}{|l|}{ HCC treatment } \\
\hline Hepatic resection & $5(16.1 \%)$ & $6(31.5 \%)$ & $x^{2}=4.989^{*}$ & $p=0.035^{*}$ \\
\hline RFA & $16(51.6 \%)$ & $11(57.8 \%)$ & $x^{2}=0.693$ & $p=0.405$ \\
\hline LDLT & $8(25.8 \%)$ & $2(10.5 \%)$ & $x^{2}=5.837^{*}$ & $p=0.018^{*}$ \\
\hline Alcohol injection & $2(6.4 \%)$ & $0(0.0 \%)$ & $x^{2}=0.625$ & $p=1.000$ \\
\hline \multicolumn{5}{|c|}{ HCV treatment (weeks) } \\
\hline Min.-Max. & $12.0-24.0$ & $12.0-24.0$ & $t=1.202$ & 0.238 \\
\hline Mean \pm SD & $13.94 \pm 4.49$ & $15.79 \pm 5.73$ & & \\
\hline Median & 12.0 & 12.0 & & \\
\hline \multicolumn{5}{|l|}{$\mathrm{HCV}$ treatment } \\
\hline SOF/DAC & $1(3.2 \%)$ & $0(0.0 \%)$ & $x^{2}=0.625$ & $p=1.000$ \\
\hline SOF/DAC/RBV & $17(54.8 \%)$ & $13(68.4 \%)$ & $x^{2}=0.905$ & $p=0.341$ \\
\hline SOF/LED & $3(9.7 \%)$ & $2(10.5 \%)$ & $x^{2}=0.009$ & $p=1.000$ \\
\hline SOF/LED/RBV & $0(0.0 \%)$ & $1(5.3 \%)$ & $x^{2}=1.665$ & $p=0.380$ \\
\hline SOF/RBV & $6(19.4 \%)$ & $3(15.8 \%)$ & $x^{2}=0.101$ & $p=1.000$ \\
\hline SOF/SIM & $4(12.9 \%)$ & $0(0.0 \%)$ & $x^{2}=2.665$ & $p=0.284$ \\
\hline \multicolumn{5}{|l|}{ PCR after TT } \\
\hline SVR & $29(93.5 \%)$ & 13 (68.4\%) & $x^{2}=5.534^{*}$ & $p=0.041^{*}$ \\
\hline Relapser & $2(6.5 \%)$ & $6(31.6 \%)$ & & \\
\hline
\end{tabular}

SOF sofosbuvir, LED ledipasvir, RBV ribavirin, SIM simeprevir, DAC daclatasvir, SVR sustained virological response

Also, these results agreed with those obtained by El Kassas et al. who found that among the 53 patients treated with DAAs, they observed $37.7 \%$ recurrence after a median of 16.0 months of follow-up. Among the 63 patients not treated with DAAs, they observed a $25.4 \%$ HCC recurrence after a median of 23.0 months of follow-up [8].

Another study by Rewisha et al. presented a case report that included a series of 16 patients who were diagnosed as Child A HCV-related cirrhosis. All patients received IFN-free, sofosbuvir-based regimens. Sofosbuvir plus ribavirin was prescribed to 11 cases $(68.8 \%)$; 14 patients had predominantly small $\mathrm{HCC}$ and an occurrence time of $4.19 \pm 3.48$ months post-treatment [21]. However, the study did not include HCC intervention.

On the other hand, several studies were consistent with our study, the report published by the French Agency for Research on AIDS and Viral Hepatitis, and the ANRS (France Recherche Nord and Sud For Hepatitis and HIV). The report included 3 ANRS cohorts which showed a lack of evidence on DAAs' effect on increased HCC recurrence rates. Where the first study showed a recurrence rate of $12.7 \%$ in treated versus $20.5 \%$ in non-treated patients, the second showed a CO23 CUPILT cohort which showed a recurrence rate of $2.2 \%$ in liver transplant recipients receiving DAA therapy. But, this study did not involve a control arm [22].

A study by Kanwal et al. reported that cirrhotic patients with SVR had a significantly reduced risk of HCC compared to patients without SVR (76\% risk reduction). They highlighted that the HCV-treated population has changed significantly in the DAA era and now includes many patients with other HCC risk factors (alcohol abuse, age older than 65 years, and patients with advanced cirrhosis), which were excluded before from IFN therapy [23].

A prospective observational study by Calvaruso et al. concluded the early benefit of viral eradication in HCV cirrhosis throughout all stages of cirrhosis. The authors analyzed only patients with cirrhosis (2249 patients: 90.5\% Child-Pugh class A, 9.5\% Child-Pugh class B) treated with DAA. Only 78 patients (3.4\%) developed HCC during a mean observation of 14 months from the start of DAA treatment; the overall cumulative rate of 
Table 5 Relation between HCC recurrence and different parameters in group ॥

\begin{tabular}{|c|c|c|c|c|}
\hline & \multicolumn{2}{|l|}{ HCC recurrence } & \multirow{2}{*}{$\begin{array}{l}\text { Test of } \\
\text { Sig. }\end{array}$} & \multirow[t]{2}{*}{$p$} \\
\hline & $\begin{array}{l}\text { No }(n=19), \text { no. } \\
(\%)\end{array}$ & $\begin{array}{l}\text { Yes }(n=31) \text {, no. } \\
(\%)\end{array}$ & & \\
\hline \multicolumn{5}{|l|}{ Focal lesion } \\
\hline 1 & $10(52.6 \%)$ & $23(74.2 \%)$ & \multirow{3}{*}{$\begin{array}{l}x^{2}= \\
2.583\end{array}$} & \multirow{3}{*}{$\begin{array}{l}p= \\
0.227\end{array}$} \\
\hline 2 & $6(31.6 \%)$ & $5(16.1 \%)$ & & \\
\hline 3 & $3(15.8 \%)$ & $3(9.7 \%)$ & & \\
\hline \multicolumn{5}{|l|}{ Overall size $(\mathrm{cm})$} \\
\hline Min.-Max. & $2.20-8.0$ & $1.30-7.50$ & \multirow{3}{*}{$\begin{array}{l}t= \\
1.653\end{array}$} & \multirow[t]{3}{*}{0.105} \\
\hline Mean \pm SD & $4.10 \pm 1.64$ & $3.40 \pm 1.32$ & & \\
\hline Median & 4.0 & 3.0 & & \\
\hline \multicolumn{5}{|l|}{$\mathrm{BCLC}$} \\
\hline 0 & $2(7 \%)$ & $0(0.0 \%)$ & \multirow[t]{3}{*}{$x^{2}=$} & \multirow[t]{3}{*}{0.075} \\
\hline A & 7 (36.8\%) & $19(61.3 \%)$ & & \\
\hline B & $10(52.6 \%)$ & 12 (38.7\%) & & \\
\hline \multicolumn{5}{|l|}{ HCC treatment } \\
\hline $\begin{array}{l}\text { Hepatic } \\
\text { resection }\end{array}$ & $7(36.8 \%)$ & $5(16.1 \%)$ & $\begin{array}{l}x^{2}= \\
3.207\end{array}$ & $\begin{array}{l}p= \\
0.049\end{array}$ \\
\hline RFA & $11(57.8 \%)$ & $24(77.4 \%)$ & $\begin{array}{l}x^{2}= \\
3.311\end{array}$ & 0.069 \\
\hline $\begin{array}{l}\text { Alcohol } \\
\text { injection }\end{array}$ & $1(5.2 \%)$ & $2(6.5 \%)$ & $\begin{array}{l}x^{2}= \\
0.543\end{array}$ & 0.461 \\
\hline
\end{tabular}

HCC at 1 year was $2.9 \%$. The occurrence of $\mathrm{HCC}$ is significantly reduced in patients with compensated cirrhosis. The authors did not support the hypothesis that HCC that develops during DAA treatment or after early follow-up is more aggressive and more difficult to treat with available therapies [24]. These results support that obtained by the present study concerning the recurrence of HCC, but the limitation in our results is the lack of data about the aggressiveness of the recurrent tumor.

The results of this study reported an $\mathrm{HCC}$ recurrence rate of $38 \%$ in patients with treated $\mathrm{HCC}$ who received DAAs compared to $62 \%$ in DAA-non-treated patients proving that clearance of HCV infection using DAA therapy did not increase the risk of $\mathrm{HCC}$ recurrence and it did not abolish it.

Table 6 HCC recurrence rate and time after HCC intervention in group I

\begin{tabular}{lll}
\hline & Number & Percent \\
\hline HCC recurrence & & \\
No & 31 & 62.0 \\
Yes & 19 & 38.0
\end{tabular}

Rec time after the start of DAAs (months) $(n=19)$

$\begin{array}{ll}\text { Min.-Max. } & 5.0-18.0 \\ \text { Mean } \pm \text { SD } & 9.47 \pm 4.14 \\ \text { Median } & 8.0\end{array}$

Table 7 HCC recurrence rate and time after HCC intervention in group II

\begin{tabular}{lll}
\hline & Number & Percent \\
\hline HCC recurrence & 19 & 38.0 \\
No & 31 & 62.0 \\
Yes & & \\
Time (months) & $1.0-12.0$ & \\
Min.-Max. & $3.34 \pm 2.53$ & \\
Mean \pm SD & 3.0 & \\
Median & &
\end{tabular}

So, a proper selection of patients enrolled for antiviral therapy and precise assessment of fibrosis prior to treatment using an accurate test like transient elastography are important. Besides, a close follow-up of the patients after the end of treatment especially those with advanced fibrosis and cirrhosis for a long duration is necessary.

\section{Conclusion}

HCC patients who did not receive direct-acting antiviral therapy after HCC intervention had a greater risk of HCC recurrence. DAAs did not increase the risk of $\mathrm{HCC}$ recurrence following $\mathrm{HCC}$ treatment; however, it did not abolish it. Close monitoring of patients after antiviral therapy is recommended.

\section{Abbreviations \\ AFP: Alpha-fetoprotein; ASU: Ain Shams University; BCLC: Barcelona Clinic of Liver Cancer; DAAs: Direct-acting antivirals; HCC: Hepatocellular carcinoma; HCV: Chronic hepatitis C virus; IFN: Interferon; INR: International normalized ratio; MRI: Magnetic resonance imaging; $\mathrm{NCCVH}$ : National Committee for the Control of Viral Hepatitis; SVR: Sustained virological response}

\section{Acknowledgements}

The authors would like to thank the team working in the Center for Treatment of Viral Hepatitis at Ain Shams University (ASU) for facilitating the collection of materials and data.

\section{Authors' contributions}

NM shared the study design, supervised the study, and submitted the manuscript. IM shared the study design, collected and analyzed the data, and wrote the manuscript. AA helped in the collection and analysis of the data and supervised the study. All authors have read and approved the final manuscript.

\section{Funding}

No funding was obtained for this study.

\section{Availability of data and materials}

All participants in the study were recruited from the Hepatology and Gastroentrology Unit, Internal Medicine Department, Ain Shams University Hospital, and from the Center for Treatment of Viral Hepatitis at Ain Shams University (ASU) (one of the National Committee for the Control of Viral Hepatitis (NCCVH) centers in Cairo), as well as the collected data.

\section{Ethics approval and consent to participate}

This study has been performed in accordance with ethical standards. Faculty of Medicine, Ain Shams University Ethical Committee approval was taken before starting the study (reference number FWA000017585). Written informed consent was obtained from all participants before enrollment in the study. 


\section{Consent for publication}

All listed authors who participated in this work have accepted to publish this work in the Egyptian Liver Journal.

\section{Competing interests}

The corresponding author conforms on behalf of all authors that there have been no involvements that might raise the question of bias in the work reported or in the conclusions stated.

Received: 9 September 2019 Accepted: 4 May 2020

Published online: 08 July 2020

\section{References}

1. Heimbach JK, Kulik LM, Finn RS, Sirlin CB, Abecassis MM, Roberts LR et al (2018) AASLD guidelines for the treatment of hepatocellular carcinoma. Hepatology. 67(1):358-380

2. Messina JP, Humphreys I, Flaxman A, Brown A, Cooke GS, Pybus OG et al (2015) Global distribution and prevalence of hepatitis C virus genotype. Hepatology 61(1):77-87

3. Kandeel A, Genedy M, El-Refai S, Funk AL, Fontanet A, Talaat M (2017 Jan) The prevalence of hepatitis C infection in Egypt 2015: implication for future policy on prevention and treatment. Liver Int 37(1):45-53

4. Elgharably A, Gomaa Al, Crossey MM, Norsworthy PJ, Waked I, TaylorRobinson SD (2016) Hepatitis C in Egypt - past, present, and future. Int J Gen Med 20(10):1-6

5. Rebecca LM, Brittney B, Bryce DS, Anthony Y, Marc P, Yngve FY (2013) Eradication of hepatitis $C$ virus infection and the development of hepatocellular carcinoma: a meta-analysis of observational studies. Ann Intern Med 158:329-337

6. Liping Z, Xiantao Z, Zongguo Y, Zhiqiang M (2013) Effect and safety of interferon for hepatocellular carcinoma: a systematic review and metaanalysis. PLoS One 8(9):e61361

7. Moon C, Jung KS, Kim DY, Baatarkhuu O, Park JY, Kim BK et al (2015) Lower incidence of hepatocellular carcinoma and cirrhosis in hepatitis $C$ patients with sustained virological response by pegylated interferon and ribavirin. Dig Dis Sci 60(2):573-581

8. El Kassas M, Funk AL, Salaheldin M, Shimakawa Y, Eltabbakh M, Jean K et al (2018 Jun) Increased recurrence rates of hepatocellular carcinoma after DAA therapy in a hepatitis C-infected Egyptian cohort: a comparative analysis. J Viral Hepat 25(6):623-630

9. Schinazi R, Halfon P, Marcellin P, Asselah T (2014) HCV direct-acting antiviral agents: the best interferon-free combinations. Liver Int 34(1):69-78

10. Conti F, Buonfiglioli F, Scuteri A, Crespi C, Bolondi L, Caraceni P et al (2016) Early occurrence and recurrence of hepatocellular carcinoma in HCV-related cirrhosis treated with direct acting antivirals. J Hepatol https://doi.org/10 1016/j.jhep.2016.06.015

11. Pugh RN, Murray-Lyon IM, Dawson JL, Pietroni MC, Williams R (1973) Transection of the oesophagus for bleeding oesophageal varices. Br I Surg 60:646-649

12. Kirk GD, Astemborski J, Mehta SH, Spoler C, Fisher C, Allen D et al (2009) Assessment of liver fibrosis by transient elastography in persons with hepatitis C virus infection or HIV-hepatitis C virus coinfection. Clin Infect Dis 48:963-972

13. Ghany MG, Strader DB, Thomas DL, Seeff LB (2009) American Association for the Study of Liver Diseases. Diagnosis, management, and treatment of hepatitis C: an update. Hepatology 49:1335-1374

14. Bürger C, Maschmeier M, Hüsing-Kabar A, Wilms C, Köhler M, Schmidt M, Schmidt $\mathrm{H}$, and Kabar L: Achieving complete remission of hepatocellular carcinoma: a significant predictor for recurrence-free survival after liver transplantation. Canadian Journal of Gastroenterology and Hepatology, Volume 2019, https://doi.org/10.1155/2019/5796074.

15. Kudo M, Arizumi T, Ueshima K, Sakurai T, Kitano M, Nishida N (2015) Subclassification of BCLC B stage hepatocellular carcinoma and treatment strategies: proposal of modified Bolondi's subclassification (Kinki Criteria). Dig Dis 33:751-758

16. Golfieri R, Bargellini I. Spreafico C, Trevisani F: Patients with Barcelona Clinic Liver Cancer Stages B and C hepatocellular carcinoma: time for a subclassification. Liver Cancer, 2019;8:78-91.

17. Morgan TR, Ghany MG, Kim HY, Snow KK, Shiffman ML, De Santo JL et al (2010) Outcome of sustained virological responders with histologically advanced chronic hepatitis C. Hepatology 52:833-844
18. Liovet JM and Villanueva A. Effect of HCV clearance with direct acting antiviral agents on HCC. Nature Reviews Gastroenterology \& Hepatology 2016; doi:10.1038/nrgastro.2016.140 Published online 1 Sep 2016.

19. Reig M, Mariño Z, Perelló C, Iñarrairaegui M, Ribeiro A, Lens S et al (2016) Unexpected early tumor recurrence in patients with hepatitis $C$ virus related hepatocellular carcinoma undergoing interferon-free therapy: a note of caution. J Hepatol 65:719-726

20. Bhamidimarri KR, Satapathy SK, Martin P (2017) Hepatitis C virus and liver transplantation. Gastroenterol Hepatol 13(4):214-220

21. Rewisha EA, Elsabaawy MM, Elshaarawy O, Abdallah A, Elsabaawy DM, Alhaddad OM (2017) Hepatocellular carcinoma following direct anti-viral for hepatitis $C$ treatment: a report of an Egyptian case series. Hepatoma Res 3: 178-181

22. ANRS: The ANRS collaborative study group on hepatocellular carcinoma. Lack of evidence of an effect of direct acting antivirals on the recurrence of hepatocellular carcinoma: data from three ANRS cohorts. J Hepatol 2016; https://doi.org/10.1016/j.jhep.2016.05.045.

23. Kanwal F, Kramer J, Asch SM, Chayanupatkul M, Cao Y, El-Serag HB (2017) Risk of hepatocellular cancer in HCV patients treated with direct-acting antiviral agents. Gastroenterology 153:996-1005

24. Calvaruso V, Cabibbo G, Cacciola I, Petta S, Madonia S, Bellia A et al (2018) Rete Sicilia Selezione Terapia - HCV (RESIST-HCV). Incidence of hepatocellular carcinoma in patients with HCV-associated cirrhosis treated with direct-acting antiviral agents. Gastroenterology. 155(2):411-421

\section{Publisher's Note}

Springer Nature remains neutral with regard to jurisdictional claims in published maps and institutional affiliations.

\section{Submit your manuscript to a SpringerOpen ${ }^{\circ}$ journal and benefit from:}

- Convenient online submission

- Rigorous peer review

- Open access: articles freely available online

- High visibility within the field

- Retaining the copyright to your article

Submit your next manuscript at $\boldsymbol{\nabla}$ springeropen.com 